\title{
DEFORMATION BEHAVIOR OF INTERMETALLICS: MODELS AND EXPERIMENTS
}

\author{
B.A. Greenberg ${ }^{1}$, M.A. Ivanov ${ }^{2}$, O.V.Antonova ${ }^{1}$, A.M. Patselov ${ }^{1}$, A. V. Plotnikov ${ }^{1}$ \\ ${ }^{1}$ Institute of Metal Physics, Ural Div., Russian Academy of Sciences, \\ Ekaterinburg, Russia \\ ${ }^{2}$ Institute of Metal Physics, National Academy of Sciences, Kiev, Ukraine
}

\begin{abstract}
A sufficiently general thermally activated mechanism for extension of dislocations in some preferential direction was proposed. This mechanism comprises a necessary step of dislocation transformations that lead to blocking. The reasons for blocking of different types of dislocations in different materials are diverse. A new concept was developed concerning the possibility of thermally activated blocking of superdislocations in the absence of external stresses. Some experiments with single crystals of $\mathrm{Ni}_{3}(\mathrm{Al}, \mathrm{Nb})$ were performed. They included no-load heating after preliminary low- or high-temperature deformation. It was found that the initial dislocation structure, which included curvilinear dislocations, transformed to a set of long rectilinear blocked superdislocations after no-load heating. The experimental results confirmed theoretical assumptions on the possibility of thermally activated transformations of superdislocations to indestructible barriers in the absence of external stresses.
\end{abstract}

\section{Introduction}

High-temperature intermetallics like $\mathrm{Ni}_{3} \mathrm{Al}$ and TiAl possess a number of unusual properties $(1,2)$ including an anomalous temperature dependence of the yield stress $\sigma_{\mathrm{y}}(T)$. This behavior is mainly due to specific features of their dislocation structure. The ability of dislocations in intermetallics for self-blocking distinguishes them from dislocations in other materials. The problem at issue is thermally activated transformations of dislocations from the glissile form to the blocked configuration when transformations do not involve other dislocations (3). A Kear-Wilsdorf barrier is the blocked form of superdislocations with the Burgers vector $<101>$ in $\mathrm{Ni}_{3} \mathrm{Al}$ and TiAl. It is known that the formation of a Kear-Wilsdorf barrier is stimulated by the anisotropy of the antiphase boundary (APB) energy, by which $\varsigma^{\prime}<\varsigma$. Here $\varsigma$ and $\varsigma^{\prime}$ denote energies per unit area of the APB in the octahedral and cube planes respectively. Either a Kear-Wilsdorf barrier or a "roof"-type configuration represents possible blocked forms of superdislocations with the Burgers vector $1 / 2<112>$ in TiAl. Blocking of a single dislocation with the Burgers vector $1 / 2<110>$ in TiAl is explained, in our opinion (4), by its localization in deep Peierls valleys extended in $<110>$ directions. This mechanism is somewhat analogous to the dislocation blocking mechanism in semiconductors. However, we deal with deep valleys for single dislocations in TiAl and for partial dislocations in semiconductors $(5,6)$.

A sufficiently general approach was developed earlier for description of the plastic deformation process (4). Many features of the deformation behavior of intermetallics could be explained in terms of this approach. However, some questions still remained unclear. By this study we tried to answer some of them, specifically:

- Is blocking of dislocations possible in the absence of external stresses? If yes, in what conditions?

- Can barriers be destructed in this case? 


\section{EXTENSION OF A DISLOCATION IN THE PREFERENTIAL DIRECTION}

A feature in common of dislocation transformations is the presence of some preferential direction, in which the dislocation has the least energy, but (or because of this) is blocked. It is stated in the foregoing that the reasons for blocking are diverse for dislocations of different types and in different materials. Only the direction of the $<101>$ type is preferential in the cases considered above. A mechanism by which a dislocation extends in the preferential direction was proposed in $(7,8)$. It can be shown that this mechanism is sufficiently general and comprises a mandatory step in dislocation transformations that lead to blocking.

Consecutive stages of the rearrangement of an initial dislocation, whose direction is close to the preferential one, are schematized in Fig. 1: the formation of a double kink (Fig. 1a) and its subsequent reorientation in the preferential direction (the heavy line in Fig. 1b). The internal structure of the dislocation changes in the preferential direction, leading to the decrease in the dislocation energy (shading in Fig. 1c). Since this process takes place at different points of the dislocation line, the initial dislocation breaks down into long blocked segments (Fig. 1d). Actually, the division of the stages shown in Fig. 1 may prove to be purely conditional. Firstly, as the kink is reoriented, the internal structure of the dislocation can change immediately, facilitating the process. Secondly, it is not improbable that from the energy viewpoint the immediate nucleation of an asymmetric kink, which contains the dislocation segment with the least energy, is more favorable than the nucleation of a double kink.

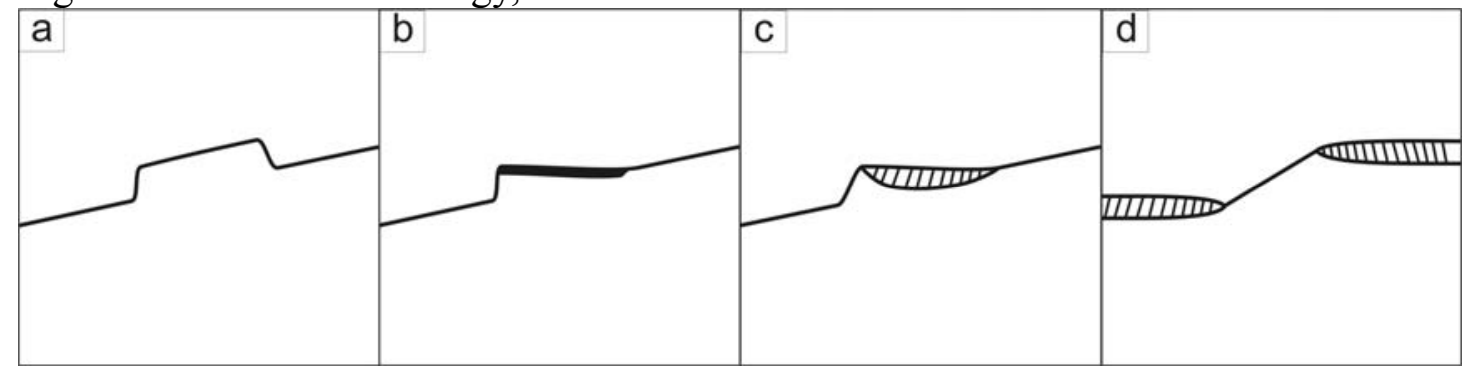

Fig. 1. Consecutive stages of the rearrangement of the initial dislocation whose direction approaches the preferential direction: $a$-double kink; $b$-re-orientation in the preferential direction; $c$ - change of the internal structure of the dislocation; $d-$ division into blocked segments

In the cases at hand, the initial dislocation is either a superpartial dislocation, which belongs to a superdislocation, or a single dislocation in intermetallics and a partial dislocation in semiconductors. Correspondingly, the change of their internal structure in intermetallics is connected either with octahedral splitting of the superpartial dislocation or, as we think, with a narrowing the single dislocation core. In semiconductors the change is due to the so-called reconstruction, which minimizes the partial dislocation core energy related to broken interatomic bonds.

In some cases, the internal structure of dislocations can change athermally. This situation will be discussed below. Generally, however, the extension of a dislocation in the preferential direction is a thermally activated process since it includes the formation of kinks and their propagation.

We shall consider, by way of example, the transformation of a superpartial dislocation from the glissile form to its blocked form when the cubic slip system operates. Here, as is customary with the formation of Kear-Wilsdorf barriers, the obvious reason for blocking of the superpartial dislocation lying in the cube plane is octahedral splitting of screw segments. Analogous splitting, which is realized for any 
segment of the dislocation loop lying in the octahedral plane in FCC metals, does not require thermal fluctuations, i.e. occurs spontaneously. But the situation is different here: octahedral splitting takes place in a plane other than the plane of location of the initial dislocation loop. Therefore, it is necessary, first and foremost, that the superpartial dislocation extends along the line of intersection between the cube and octahedral planes as shown in Fig. 1. It is only this process that exhausts the superdislocation rearrangement. Blocking of superdislocations (no-load heating after high-temperature deformation, see the corresponding section), points to the thermal activation character of this process.

A dislocation extends in the preferential direction in accordance with the scheme in Fig. 1 also in cases when dislocation transformations are not connected with the transition from one plane to another plane, but are initiated by changes in the internal structure of the dislocation core, for example, a single dislocation in TiAl and partial dislocations in semiconductors. The only thermally activated process, which determines the motion of dislocations in semiconductors (the transition from one valley to another valley), was considered earlier $(5,6)$. It was assumed implicitly that the dislocation segment extended along the Peierls valley. We think however that the segment extension should be preceded by the thermally activated extension of the partial dislocation in the preferential direction and its sinking to a deep valley.

Thus, the extension of a dislocation in the preferential direction is realized by thermal fluctuations. It still remains unclear however if and when this process can take place in the absence of the external load. Therefore, in what follows we shall calculate the effective force facilitating the process, which includes (or does not include) the contribution from the external load, for superdislocations located in the octahedral or cube plane. Two cases will be considered correspondingly: dislocation sources operate either in octahedral planes $\left(T<T_{\max }\right)$ or cube planes $\left(T>T_{\max }\right)\left(T_{\max }\right.$ is the temperature of the yield stress peak).

\section{DISLOCATION TRANSFORMATIONS (THE PRIMARY OCTAHEDRAL SLIP SYSTEM)}

\section{The role of the trailing dislocation.}

Let a superdislocation with the Burgers vector $2 \mathbf{b}$ be located in the octahedral plane (with the normal n) and consist of superpartial dislocations, which are connected with an APB band having the energy s. One of the superpartial dislocations (the leading one) is fixed by some means (for example, by jogs), while the other superpartial dislocation (the trailing one) is mobile. We shall assume that a constricted (unsplitted) segment with the Burgers vector $\mathbf{b}$ appears in the leading dislocation along the line of intersection between the octahedral and cube planes. The force $K_{(\mathbf{b}, \mathbf{n})}^{\text {eff }}$ in the octahedral plane and the force $K_{\left(\mathbf{b}, \mathbf{n}^{\prime}\right)}^{\text {eff }}$ in the cube plane act per unit length of the constricted segment near and perpendicular to this intersection line. These forces have the form

$$
\begin{gathered}
K_{(\mathbf{b}, \mathbf{n})}^{e f f}=b \sigma_{\mathbf{b n}}+\frac{\psi(\mathbf{b}, \mathbf{b})}{d}-\varsigma, \\
K_{\left(\mathbf{b}, \mathbf{n}^{\prime}\right)}^{e f f}=b \sigma_{\mathbf{b n}^{\prime}}+\alpha \frac{\psi(\mathbf{b}, \mathbf{b})}{d}-\varsigma^{\prime}, \\
\sigma_{\mathbf{b n}}=\sigma_{0} f(\mathbf{b}, \mathbf{n}), \\
\sigma_{\mathbf{b n}^{\prime}}=\sigma_{0} f\left(\mathbf{b}, \mathbf{n}^{\prime}\right),
\end{gathered}
$$




$$
\begin{gathered}
d=\psi(\mathbf{b}, \mathbf{b}) /\left(\varsigma^{+}+b \sigma_{\mathbf{b n}}\right), \\
\psi\left(\mathbf{b}_{1}, \mathbf{b}_{2}\right)=\frac{\mu}{2 \pi(1-v)}\left(\mathbf{e}_{1} \mathbf{e}_{2}+(1-v) \mathbf{s}_{1} \mathbf{s}_{2}\right) .
\end{gathered}
$$

Here $d$ is the equilibrium width of the superdislocation in the octahedral plane; $\sigma_{\mathbf{b n}}$ and $\sigma_{\mathbf{b n}^{\prime}}$ denote components of the external stress; $\sigma_{0}$ is the external axial stress; $f(\mathbf{b}, \mathbf{n})$ and $f\left(\mathbf{b}, \mathbf{n}^{\prime}\right)$ are Schmid factors; e and $\mathbf{s}$ are edge and screw components of the Burgers vector respectively; $\mu$ is the shear modulus; $v$ is the Poisson ratio; and $\alpha=\mathbf{n n}^{\prime}=1 / \sqrt{3}$. The quantity $\psi\left(\mathbf{b}_{1}, \mathbf{b}_{2}\right)$ is a coefficient in the expression for the elastic interaction force of dislocations. The subscripts in the above expressions denote slip systems.

The first term on the right of each of the equations (1) and (2) describes the force of external stresses, the second term gives the force of the elastic field of the trailing dislocation, and the third term describes the APB surface tension. Substituting (3)-(5) into (1) and (2), we have

$$
\begin{gathered}
K_{(\mathbf{b}, \mathbf{n})}^{\text {eff }}=2 b \sigma_{0} f(\mathbf{b}, \mathbf{n}) \\
K_{\left(\mathbf{b}, \mathbf{n}^{\prime}\right)}^{\text {eff }}=b \sigma_{0}\left(f\left(\mathbf{b}, \mathbf{n}^{\prime}\right)+\alpha f(\mathbf{b}, \mathbf{n})\right)+\alpha \varsigma-\varsigma^{\prime} .
\end{gathered}
$$

It is seen from (7) and (8) that $K_{\left(\mathbf{b}, \mathbf{n}^{\prime}\right)}^{\text {eff }}$ includes two parts: one part depends on $\sigma_{0}$ similarly to $K_{(\mathbf{b}, \mathbf{n})}^{\text {eff }}$, while the other part is independent of $\sigma_{0}$. The expression (7) reflects the fact that the stress concentration $\left(n \sigma_{0}\right)$ takes place at the head of a pileup comprising $n$ (in this case, two) dislocations. The term $\alpha_{\varsigma}$ in (8) is due to $\alpha \psi(\mathbf{b}, \mathbf{b}) / d$, i.e. the cube plane projection of the radial force of the elastic repulsion acting on the leading dislocation from the trailing dislocation.

When one compares (7) and (8), he should take into account that generally the following relation is fulfilled:

$$
\varsigma / b>\sigma_{0},
$$

which actually represents the condition for the plastic deformation by superdislocations (rather than by superpartial dislocations). The comparison of experimental values of $\varsigma / b$ and $\sigma_{0}$ shows that in standard conditions $\varsigma / b$ is several times and, in some cases, one order of magnitude larger than $\sigma_{0}$. Using typical values of $\varsigma \cong 200-300 \mathrm{~mJ} / \mathrm{m}^{2}$ and $b \cong 0.2 \mathrm{~nm}$ [1], we obtain $\varsigma / b \cong(1-1.5) \cdot 10^{3} \mathrm{MPa}$. Since $\sigma_{0}$ $\cong(1.5-3) \cdot 10^{2} \mathrm{MPa}$, we make certain that the relation (9) holds.

Thus, it follows from (7) and (8) that the difference of $K_{(\mathbf{b}, \mathbf{n})}^{\text {eff }}$ and $K_{\left(\mathbf{b}, \mathbf{n}^{\prime}\right)}^{\text {eff }}$ is determined mostly by $\Delta=\alpha \varsigma-\varsigma^{\prime}$, which is independent of $\sigma_{0}$. The term $\alpha \varsigma$ facilitates and the term $\varsigma^{\prime}$ inhibits bending of the constricted segment to the cube plane.

Blocking of dislocations at $\sigma_{0}=0$.

In this case, the effective force in the cube plane $K_{\left(\mathbf{b}, \mathbf{n}^{\prime}\right)}^{\text {eff }}$ remains nonzero, as distinct from $K_{(\mathbf{b}, \mathbf{n})}^{\text {eff }}$. In accordance with (2) and (5) it is defined by the expression

$$
K_{\left(\mathbf{b}, \mathbf{n}^{\prime}\right)}^{e f f}=\alpha \frac{\psi(\mathbf{b}, \mathbf{b})}{d}-\varsigma^{\prime}=\alpha \varsigma-\varsigma^{\prime},
$$




$$
K_{(\mathbf{b}, \mathbf{n})}^{\text {eff }}=0
$$

In our opinion, it is the force $\alpha \psi(\mathbf{b}, \mathbf{b}) / d$, which acts from the trailing dislocation on the leading dislocation and stimulates its transition to the cube plane, that makes possible the formation of barriers in the absence of external stresses. It is seen from (10) that the relationship

$$
\Delta=\alpha \varsigma-\varsigma^{\prime}>0
$$

is the condition for $K_{\left(\mathbf{b}, \mathbf{n}^{\prime}\right)}^{\text {eff }}>0$ at $\sigma_{0}=0$.

As a result, the assistance of the trailing dislocation makes possible the generation of double kinks and their propagation in the cube plane even in the absence of the load. Clearly this process requires the thermal activation in any event. But first a constriction should be formed in the split superpartial dislocation. However, at $\sigma_{0}=0$ constrictions cannot appear because $K_{(\mathbf{b}, \mathbf{n})}^{\text {eff }}=0$. Then the superpartial dislocation should have "ready" constrictions. These constrictions can be formed, for example, when dislocations cross the "forest" during preliminary deformation at a low temperature.

The above theoretical analysis suggests that thermally activated blocking of dislocations in the absence of external stresses is possible if the following conditions are fulfilled:

- initial dislocations have ready constrictions;

- the relation $\alpha \varsigma>\varsigma^{\prime}$ between the APB energies in the octahedral and cube planes holds.

Analogous calculations were performed to ascertain how the elastic interaction of superpartial dislocations influences the reverse transformation of the blocked configuration to the glissile form (the barrier destruction). It was shown that the effect of the trailing dislocation on the cross-slip of the leading dislocation was much weaker in the case of the barrier destruction than during its formation. Moreover, at $\sigma_{0}=0$ the corresponding effective force was zero unlike the force $K_{\left(\mathbf{b}, \mathbf{n}^{\prime}\right)}^{\text {eff }}$. This means that barriers are formed, but are not destructed during no-load heating.

\section{DISLOCATION TRANSFORMATIONS (THE PRIMARY CUBIC SLIP SYSTEM)}

At $T>T_{\max }$ the plastic deformation ( $\dot{\varepsilon}=$ const) is realized by the cubic slip (1, $2,4)$. Similarly to the previous case, in this case the process responsible for blocking of the superpartial dislocation is the same extension of the segment in the preferential $<101>$ direction. However, while in the previous case the initial superpartial dislocation is split in the octahedral plane, now the initial superpartial dislocation, which is located originally in the cube plane, is not split. Hence, the rearrangement is limited only by the extension of the segment in the said direction, including octahedral splitting of the superpartial dislocation.

It is this case that was considered above as an example in the discussion of the consecutive stages of the rearrangement (Fig. 1). It should be emphasized that the trailing partial dislocation remains fixed at the above-mentioned line of intersection. This situation arises because the inequality (9) is fulfilled. The emission of the leading partial dislocation in the octahedral plane is facilitated by the mutual repulsion of the 
partial dislocations. Since octahedral splitting is athermal, one may expect blocking of the superdislocation at a zero external stress. Oppositely, the reverse process unblocking includes the recombination of the superpartial dislocation, which requires external stresses. Therefore, the barriers, which are considered here, also remain indestructible upon no-load heating.

\section{OBSERVATION OF BLOCKED SUPERDISLOCATIONS UPON NO-LOAD HEATING}

The subjects of the experimental study were $\mathrm{Ni}_{3}(\mathrm{Al}, \mathrm{Nb})$ single crystals, which were grown by the Bridgman method and were homogenized at $1300^{\circ} \mathrm{C}$ for 100 hours. Samples in the form of prisms with the square section of $3.3 \mathrm{~mm}$ and $8 \mathrm{~mm}$ high were cut out by the electric spark method using a goniometric head for the X-ray analysis of their orientation. The samples were deformed by upsetting to $2-3.5 \%$ at a rate of $0.5 \mathrm{~mm} / \mathrm{min}$. Then they were heated without a load at different temperatures and were cooled in air. The orientation of the samples was determined from the X-ray analysis by the Laue method. The dislocation structure was examined in a JEM 200CX transmission electron microscope. Dislocations were identified by the gb analysis method.

The orientation [ $\overline{2} 51]$ was chosen such as to exclude multiple slip [9]. The system DI ([011] and (1 $\overline{1} 1))$ has the maximum Schmid factor $(f=0.490)$. The next largest Schmid factor $(f=0.435)$ is in the octahedral system DII $([01 \overline{1}]$ and $(\overline{1} 11)]$.

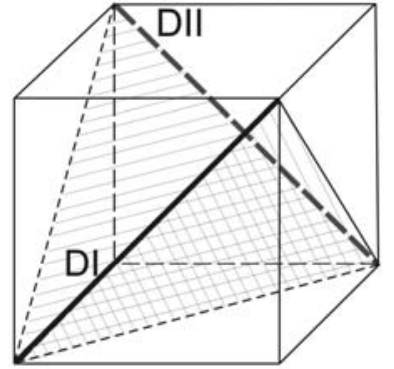

a

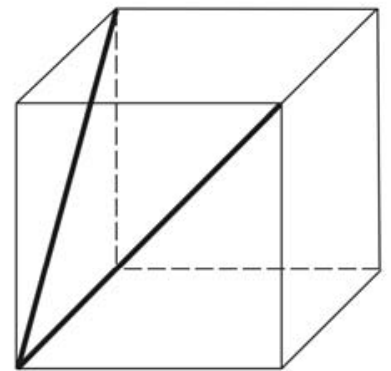

b

Fig. 2. Primary octahedral (a) and cubic (b) slip systems

The Burgers vectors in these slip systems are parallel to the cube face diagonals (100), which are marked in Fig. 2a. The $\{111\}$ slip planes, which correspond to the systems DI and DII, are shown by hatching. The (100) plane is the cross slip plane relative to these slip planes. The largest Schmid factors of 0.354 and 0.283 in cubic slip systems correspond to $[10 \overline{1}](010)$ and [011](100] slip systems. Directions of the Burgers vectors in the last systems are shown in Fig. $2 b$.

\section{Low-temperature deformation and subsequent no-load heating.}

Single crystals of $\mathrm{Ni}_{3}(\mathrm{Al}, \mathrm{Nb})$ with the [ $\left.\overline{2} 51\right]$ orientation were deformed at $-196{ }^{\circ} \mathrm{C}$. The microphotographs in Fig. 3a show the dislocation structure, which was observed after low-temperature (LT) deformation. This dislocation structure, which is typically formed at low temperatures, includes curvilinear dislocations in the octahedron planes. Then the samples were heated at $200{ }^{\circ} \mathrm{C}$ for $20 \mathrm{~min}$ and $\left(500{ }^{\circ} \mathrm{C}, 1\right.$ $\mathrm{h}+800{ }^{\circ} \mathrm{C}, 1 \mathrm{~h}$ ). The corresponding microphotographs are given in Figs. 3b, c. The dislocation structure changes drastically after no-load heating: long blocked rectilinear 
segments are observed in all the cases. It is seen that barriers have mutually perpendicular axes, which are located in the (100) planes. Out of directions of this type, we immediately take the (100) cube face diagonals because they are located simultaneously in the octahedral and cube planes. The gb analysis demonstrated that blocked dislocations indeed were screw dislocations. This implies that initial dislocations with these Burgers vectors belong to the slip systems DI and DII (Fig. 2a). Therefore, the two systems were active during LT deformation.
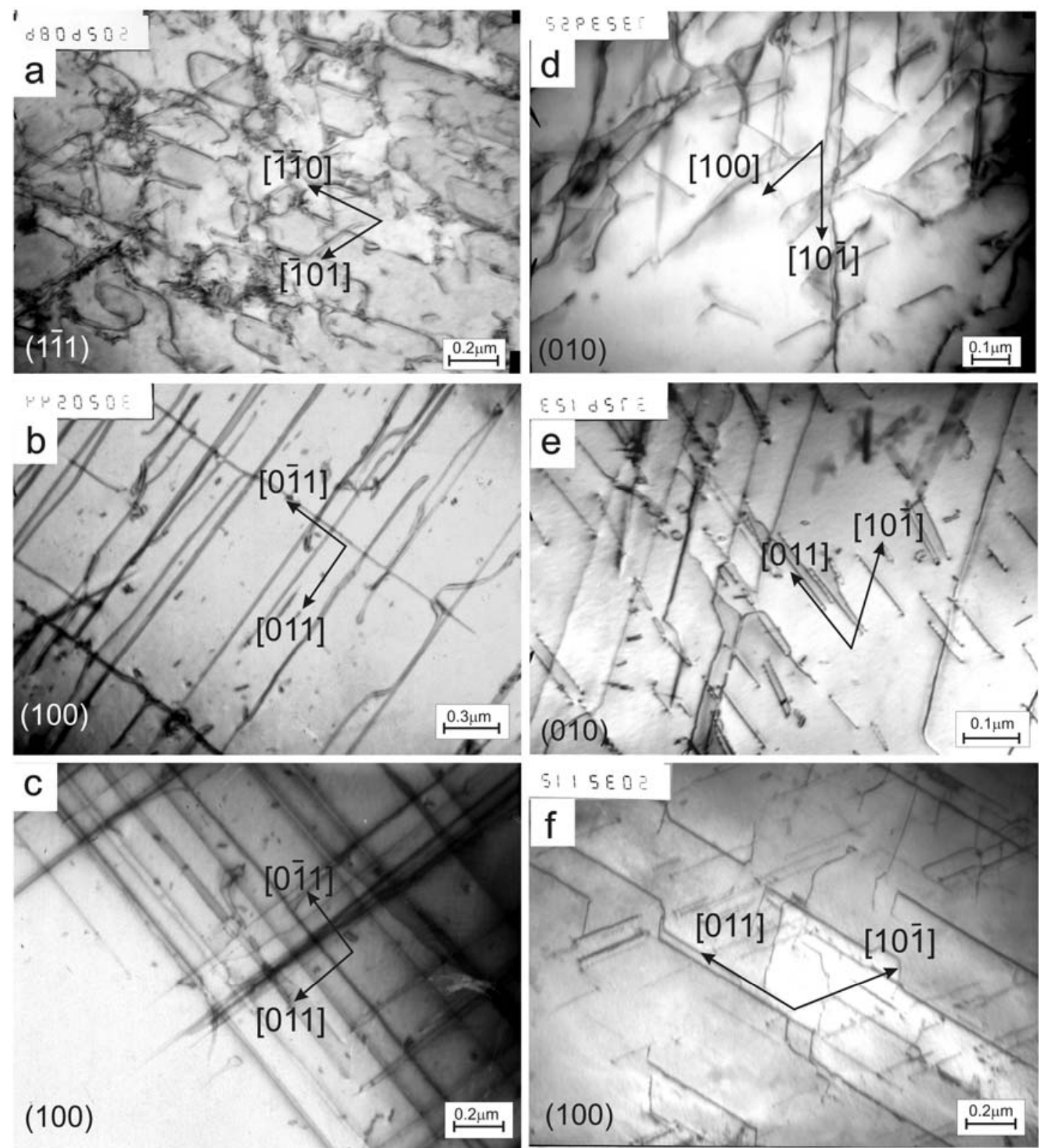

Fig. 3. Dislocation structure of $\mathrm{Ni}_{3}(\mathrm{Al}, \mathrm{Nb})$ single crystals with the [ $\left.\overline{2} 51\right]$ orientation after preliminary deformation and subsequent no-load heating: a - deformation at 77

$\mathrm{K} ; \mathrm{b}$ - heating at $200^{\circ} \mathrm{C}, 20 \mathrm{~min} ; \mathrm{c}-$ heating at $\left(500^{\circ} \mathrm{C}, 1 \mathrm{~h}+800^{\circ} \mathrm{C}, 1 \mathrm{~h}\right) ; \mathrm{d}-$ deformation at $800{ }^{\circ} \mathrm{C}$; e, f-heating at $200{ }^{\circ} \mathrm{C}, 1 \mathrm{~h}$

\section{High-temperature deformation and subsequent no-load heating.}

Single crystals of $\mathrm{Ni}_{3}(\mathrm{Al}, \mathrm{Nb})$ with the $[\overline{2} 51]$ orientation were deformed at 800 ${ }^{\circ} \mathrm{C}$ and were quenched. The microphotograph in Fig. 3d shows the observed dislocation structure. This dislocation structure, which is characteristic of high- 
temperature (HT) deformation, consists of curvilinear dislocations in cube planes. Long blocked rectilinear segments were observed after subsequent heating $\left(200{ }^{\circ} \mathrm{C}, 1\right.$ h). Bright-field images of dislocations in the (010) and (100) planes are given in Figs. 3e, f. Directions of their axes and Burgers vectors were determined. These directions are [10 $\overline{1}]$ and [011], which represent axes of the observed barriers. This means that the initial dislocations have the same Burgers vectors and belong to the aforementioned slip systems with the largest Schmid factors among the cubic systems (Fig. 2b).

\section{DISCUSSION OF TEM RESULTS}

Dislocation sources cannot operate during no-load heating and, for this reason, the initial dislocation structure is inherited. Our study also showed that the initial structure transformed to a set of barriers. It should be emphasized that the no-load heating time was much longer (more than one order of magnitude) than the time used for dynamic loading. Nevertheless, the barriers remained indestructible.

It is known that the temperature peak of the yield stress $\sigma_{y}(T)$ is observed during active loading ( $\dot{\varepsilon}=$ const $)$ of $\mathrm{Ni}_{3}(\mathrm{Al}, \mathrm{Nb})$ single crystals. The peak temperature $T_{\max }$ varies over the interval of $750-950{ }^{\circ} \mathrm{C}$ [10] and $750-1150{ }^{\circ} \mathrm{C}$ (11) depending on the orientation. The no-load heating temperatures, which were used after LT deformation in this study, were knowingly lower or on the contrary higher than $T_{\max }$ : $200{ }^{\circ} \mathrm{C}$ and $800{ }^{\circ} \mathrm{C}$ respectively. Still, whichever the heating temperature, the dislocation structure proved to be similar to the one, which was observed only in the region of the anomalous trend of $\sigma_{y}(T)$ in the case of active loading. This means that barriers were formed, but were not destructed in the both cases.

It is seen from Figs. 3e, $\mathrm{f}$ that in the case of HT deformation and subsequent no-load heating, barriers were formed, but were not destructed. In this case, however, axes of the barriers were located in primary cubic slip planes. It should be noted that at the [251] orientation used here, barriers with the [011] axis were observed in both cases. Barriers with the [101] axes were observed only in the latter case and barriers with the $[01 \overline{1}]$ axes were formed in the former case. A feature in common was that the barriers were observed during no-load heating over the whole temperature interval.

It follows from the TEM analysis that the dislocation structure, which appeared after no-load heating, did not replicate the structure observed after dynamic loading.

\section{POSSIBLE APPLICATIONS OF THE NO-LOAD HEATING EXPERIMENTS}

These experiments can be used, firstly, as a method for identification of dislocations induced by preliminary cold deformation. The observation of dislocations in the blocked form allows discriminating them against the background of all the dislocation structure. Therefore, the identification of dislocations is considerably simplified.

Secondly, the experiments can reveal "latent" dislocation transformations, which are unobservable after dynamic loading. Being indestructible, the corresponding barriers can be detected after no-load heating. Barriers, which were formed and destructed during the cubic slip after dynamic loading, were observed in experiments with HT deformation. 
Thirdly, the experiments may be used as a rapid method for detection of the anomaly in $\sigma_{\mathrm{y}}(T)$. To understand if this anomaly is observed in some alloy, it will suffice to deform its sample at a low temperature and then heat it at a high temperature. If blocked dislocations are observed, the alloy may be expected to have anomalous $\sigma_{\mathrm{y}}(T)$ over some temperature interval. Indeed, if any thermally activated mechanism shows itself during no-load heating, it will all the more reveal itself during plastic deformation.

We performed experiments on no-load heating after preliminary deformation so as to ascertain if transformations of superdislocations to barriers can be expected and, hence, if the anomaly of $\sigma_{y}(T)$ can be observed in the $\mathrm{Ni}_{3} \mathrm{Fe}$ alloy. The temperature dependence $\sigma_{\mathrm{y}}(T)$ (12) for the ordered $\mathrm{Ni}_{3} \mathrm{Fe}$ alloy exhibited the following behavior. The yield stress $\sigma_{\mathrm{y}}$ decreased over a wide temperature interval as the temperature was elevated and increased near the ordering temperature $T_{\mathrm{c}}$ equal to about $500{ }^{\circ} \mathrm{C}$. This rise of $\sigma_{\mathrm{y}}(T)$ is also observed in some other ordered alloys and is connected with dislocation inhibition mechanisms, which are due to the decrease in the degree of the long-range order and, hence, the drop of the APB energy near $T_{\mathrm{c}}$. These inhibition mechanisms have nothing to do with the transformations of dislocations to barriers considered above. A weak peak near $300^{\circ} \mathrm{C}$ can be observed in the $\sigma_{\mathrm{y}}(T)$ curve (12). A similar peak near the same temperature is seen in the $\sigma_{\mathrm{y}}(T)$ curve in Ref. (13). In both cases the peak is observed before the aforementioned rise of $\sigma_{\mathrm{y}}(T)$.
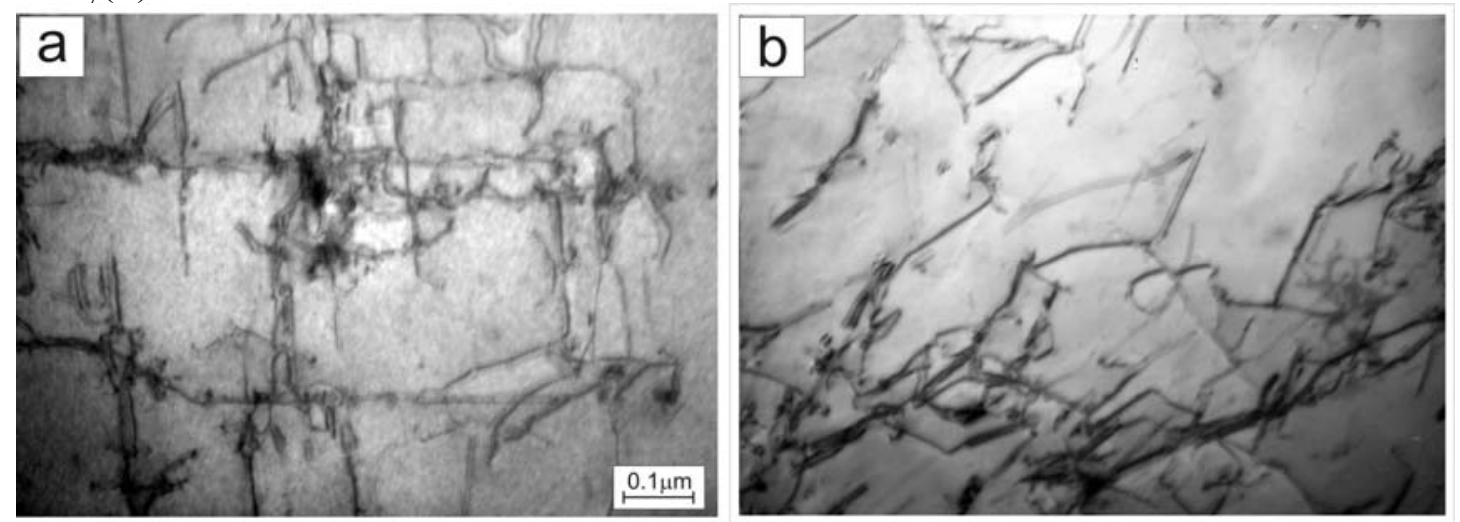

Fig. 4. Dislocation structure of the ordered $\mathrm{Ni}_{3} \mathrm{Fe}$ alloy after preliminary deformation and subsequent no-load heating: a - deformation at $-196^{\circ} \mathrm{C}$; b - heating at $200{ }^{\circ} \mathrm{C}, 1 \mathrm{~h}$

Experiment was made to ascertain if the $\sigma_{\mathrm{y}}(T)$ peak in $\mathrm{Ni}_{3} \mathrm{Fe}$ has the same origin as in $\mathrm{Ni}_{3} \mathrm{Al}$. It included ordering annealing at temperatures of $465-485{ }^{\circ} \mathrm{C}$ for 100 hours in evacuated quartz ampoules, deformation of samples by upsetting at the liquid nitrogen temperature to $4-6 \%$ at a rate of $0.5 \mathrm{~mm} / \mathrm{min}$, no-load heating at different temperatures, and cooling in air. It is seen from the microphotographs in that the dislocation structure changed insignificantly. However, the relative degree of the long-range order, which was determined by a standard X-ray method [14], was not larger than 0.77. Since the APB energy has a quadratic dependence on the degree of the long-range order, the effective force $K_{\left(\mathbf{b}, \mathbf{n}^{\prime}\right)}^{\text {eff }}$, which is defined by (10) and facilitates blocking of dislocations, is sensitive to whether ordering is complete or partial. To establish finally the absence of the anomalous $\sigma_{\mathrm{y}}(T)$ in $\mathrm{Ni}_{3} \mathrm{Fe}$, we shall perform experiments on samples with a larger degree of the long-range order. 


\section{Conclusion}

It was shown that a mandatory component of dislocation transformations that lead to blocking is a sufficiently general process of the extension of a dislocation in the preferential direction, in which the dislocation has the minimum energy.

A new concept was proposed concerning the possibility of thermally activated blocking of superdislocations in the absence of external stresses. For verification of this concept it was proposed to make experiments on no-load heating after preliminary deformation of intermetallics.

The experiments demonstrated that no-load heating of $\mathrm{Ni}_{3}(\mathrm{Al}, \mathrm{Nb})$ single crystals after preliminary low- and high-temperature deformation led to a radical change of the dislocation structure, which was caused by blocking of dislocations. It was shown that one of the dislocations (the partial or superpartial one), which was part of a superdislocation, stimulated the formation of barriers during no-load heating.

This study was financed by the RFBR (grant No. 03-02-16315).

\section{REFERENCES}

1. Veyssier P., Saada G.:'Microscopy and Plasticity of the L12 Phase'. Dislocations in Solids. Amsterdam, Elsevier 199610255.

2. Viguer B., Martin J.L., Bonneville J.:'Work Hardening in Some Ordered Intermetallic Compounds'.Dislocations in Solids. Amsterdam, Elsevier 200211 460-545.

3. Escaig B.: 'Dislocation Splitting and the Plastic Glide Process in Crystals'. J. Phys. C7. 1974. Vol. 35. P, 151-166.

4. Greenberg B. A. and Ivanov M. A.: Microstructure and Deformation Behavior of Ni3Al and TiAl Intermetallic Compounds. Ekaterinburg, Ural. Otd. Ross. Akad. Nauk, 2002 [in Russian].

5. Alexander H.: 'Dislocations in Covalent Crystals'. Dislocations in Solids. Amsterdam, Elsevier Sci. Publ., 19867 113-234.

6. Rabier J., George A.: 'Dislocations and plasticity in semiconductors'. Revue Phys. Appl. 198722 (11) 1327-1351.

7. Greenberg B.A., Ivanov M.A.: 'Some features of formation and destruction of dislocation barriers in intermetallics. I. Theory'. The Physics of Metals and Metallography 2006101 (1), (to be published).

8. Greenberg B.A., Antonova O.V., Ivanov M.A., Patselov A.M., Plotnikov A.V.: 'Some features of formation and destruction of dislocation barriers in intermetallics. II. Observation of blocking superdislocations after no-load heating'. The Physics of Metals and Metallography 2006101 (1), (to be published).

9. Inui H., Matsumuro M., Wu D.H. and Yamaguchi M.: 'Temperature dependence of yeild stress, deformation mode and deformation structure in single crystals of TiAl (Ti56 at . \%Al)'. Phil. Mag. A 199775 (2) 395-423.

10. Lall C., Chin S., Pope D.P. : 'The Orientation and Temperature Dependence of the Yield Stress of Ni3(Al,Nb) Single Crystals'. Metall. Trans. A. 1979 10a (9) 1323-1332.

11. Ezz S.S., Pope D.P., Paidar V.: 'The Tension/Compression flow stress asymetry in Ni3(A1,Nb) Single Crysrala'. Acta Met. 198230921 - 926.

12. Wee D. M., Suzuki T.: 'Temperature dependence of the yield stress of Ni3Fe single crystals'. Trans. JIM. 198122 (3) 163-172.

13. Starenchenko V.A., Solovieva Yu.V., Starenchenko S.V., Kovalevskaya T.A.: 'Thermal and deformational strengthening for monocrystals of L12 alloys'. Tomsk, NTL, 2006 [in Russian].

14. Morris D.G., Brown G.T., Piller R.C. and Smallman R.E.: 'Ordering and domain growth in Ni3Fe'. Acta Met. 197624 21-28. 